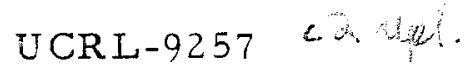

Limited Distribution

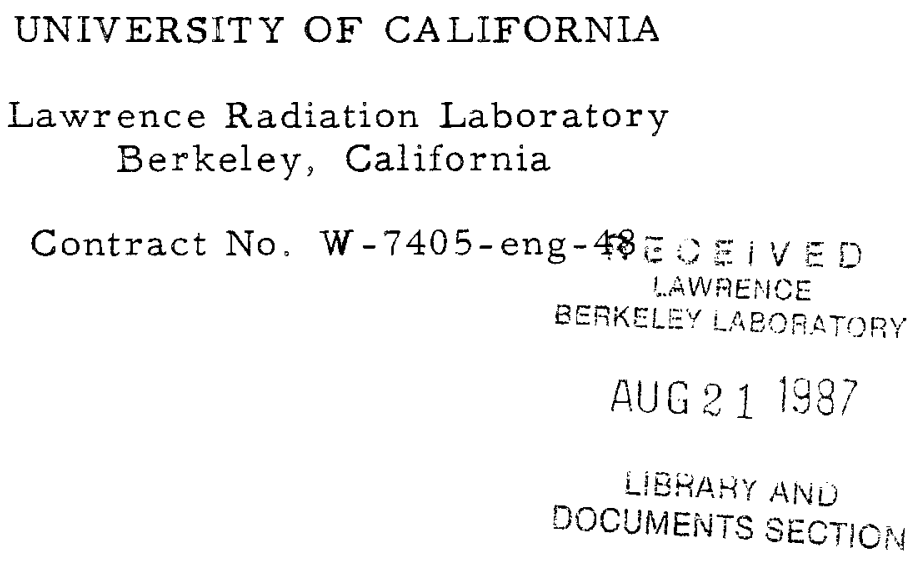

FISSION OF GOLD BY CARBON IONS

Glen E. Gordon, Almon E. Larsh, Torbjørn Sikkeland, and Glenn T. Seaborg

June 8, 1960

TWO-WEEK LOAN COPY

This is a Library Circulating Copy

which may be borrowed for two weeks. 


\title{
FISSION OF GOLD BY CARBON IONS
}

\author{
Glen E. Gordon, Almon E. Larsh, \\ Torbjørn Sikkeland, and Glenn T. Seaborg \\ Lawrence Radiation Laboratory and Department of Chemistry \\ University of California, Berkeley, California \\ June 8, 1960
}

\begin{abstract}
Angular distribution and kinetic-energy spectra of fragments, and cross sections for fission of gold with 68 - to $124-\mathrm{Mev} \mathrm{C}^{12}$ ions have been obtained by observation of the fragments in two types of detectors, gas scintillation chambers and silicon $\mathrm{p}-\mathrm{n}$ junctions. From the parameters used to fit the angular distributions to the theoretical curves of Halpern and Strutinski, we have obtained the average excitation energy of the fissioning nucleus at the time of fission. This quantity is approximately $25 \mathrm{Mev}$, which is nearly independent of bombarding energy, suggesting that fission is preceded by the emission of several particles from the compound nucleus. The fission cross section increases from a value of $100 \mathrm{mb}$ at $68 \mathrm{Mev}$ to $1.28 \mathrm{~b}$. at $124 \mathrm{Mev}$. Over this range of bombarding energies, the total fragment kinetic-energy release rises from $142 \pm 6$ to $146 \pm 6 \mathrm{Mev}$. At all bombarding energies, the variation of laboratory-system kinetic energy of the fragments with laboratory-system angle indicates full momentum transfer by the bombarding particle to the fissioning system.
\end{abstract}




\title{
FISSION OF GOLD BY CARBON IONS ${ }^{*}$
}

\author{
Glen E. Gordon, ${ }^{\dagger}$ Almon E。 Larsh, \\ Torbjørn Sikkeland, and Glenn T. Seaborg \\ Lawrence Radiation Laboratory and Department of Chemistry \\ University of California, Berkeley, California \\ June 8,1960
}

\section{INTRODUCTION}

In 1955, A. Bohr proposed a model which explained the then existing data on angular distributions of fragments from low-energy photonand neutron-induced fission of heavy elements. ${ }^{1}$ More recently, Griffin ${ }^{2}$ and, independently, Halpern and Strutinski ${ }^{3}$ have extended Bohr's model to include fission induced by higher-energy particles. The treatment used by the latter authors has been rather successful in explaining the angular distributions of fragments from $43-\mathrm{Mev} \mathrm{He}^{4}$-induced fission of several heavy elements. ${ }^{4}$ In this work it was of interest to test these theoretical treatments by studying the fission of nuclei formed with extremely large angular momenta by heavy-ion bombardment. For large values of the angular momentum of the compound nucleus $(\vec{I})$ and small values of the projection of $\vec{I}$ along the direction of the separating fragments, Halpern and Strutinski's model predicts angular distributions that follow a $1 / \sin \theta$ curve in the region around 90 deg (centerof-mass system) and fall below $1 / \sin \theta$ near 0 and $180 \mathrm{deg}$. Griffin's predicted angular distributions are similar but in some cases go above the $1 / \sin \theta$ curve.

\footnotetext{
This work was done under the auspices by the U. S. Atomic Energy Commission.

$\dagger$ Present address: Department of Chemistry, Massachusetts Institute of Technology, Cambridge, Mass.
} 
The parameters obtained by fitting the experimental angular distributions with theoretical curves were used to estimate the average excitation energy of the fissioning nucleus at the time of fission. By obtaining this quantity, one is able to determine an average number of particles emitted prior to fission. Fairhall et al. have interpreted their results as indicating that for compound nuclei with $\mathrm{z}<90$ produced in helium-ion bombardments, $\Gamma_{f} / \Gamma_{\mathrm{n}}$ (ratio of level width for fission to that for neutron emission) increases with excitation energy at least to approximately $35 \mathrm{Mev}^{5}$ Therefore, they suggest that most of the fission observed from the compound nuclei at these excitation energies must occur before neutron evaporation reduces the excitation energy and fission probability.

In addition to the angular distributions, we have also obtained information on the total fragment kinetic-energy release and on the cross sections for fission of gold with carbon ions at energies between 68 and $124 \mathrm{Mev}$. The results of some of the early phases of this study have been reported elsewhere. 6 


\section{EXPERIMENTAL PROCEDURES}

Carbon-ion beams were obtained from the Berkeley heavy-ion linear accelerator (Hilac), a resonant-cavity machine that accelerates heavy ions to $10.4 \mathrm{Mev} /$ nucleon. Angular-distribution experiments were performed in the vacuum tank shown in Fig. 1. During the experiments, the tank was connected to the Hilac vacuum system in which pressures were of the order of $5 \times 10^{-6} \mathrm{~mm} \mathrm{Hg}$. The targets, consisting of approximately $200 \mu \mathrm{g} / \mathrm{cm}^{2}$ of gold vaporized onto $0.1-\mathrm{mil}$ aluminum backing foil, were oriented at $45 \mathrm{deg}$ to the beam, with the gold facing toward or away from the beam when fragments were observed at backward or forward angles, respectively. The energy of the carbon ions was varied by placing aluminum degrading foils in the beam path ahead of the vacuum tank. The resulting particle energies were determined by use of the range-energy curves of

J. R. Walton. ${ }^{7}$ Before striking the target, the beam passed through two 1/8-in. diam. collimators, 4 in. apart. Beam particles were collected in a Faraday cup at the rear of the vacuum tank. A simpler arrangement, in which a gas scintillation counter was fixed at 90 deg to the beam, was used for experiments in which it was necessary to observe fragments only at $90 \mathrm{deg}$ to the beam.

The gas-scintillation technique has been discussed by several

authors. $8-10$ Fragments entered the gas scintillation chamber through a 0.03-mil nickel window, which was supported by a grid that transmitted $49 \%$ of the impinging particles. The scintillating gas, argon, was flushed through the chamber at 1 atmos. "Tygon" paint and diphenyl stilbene served as the reflector and wave-length shifter, respectively. 


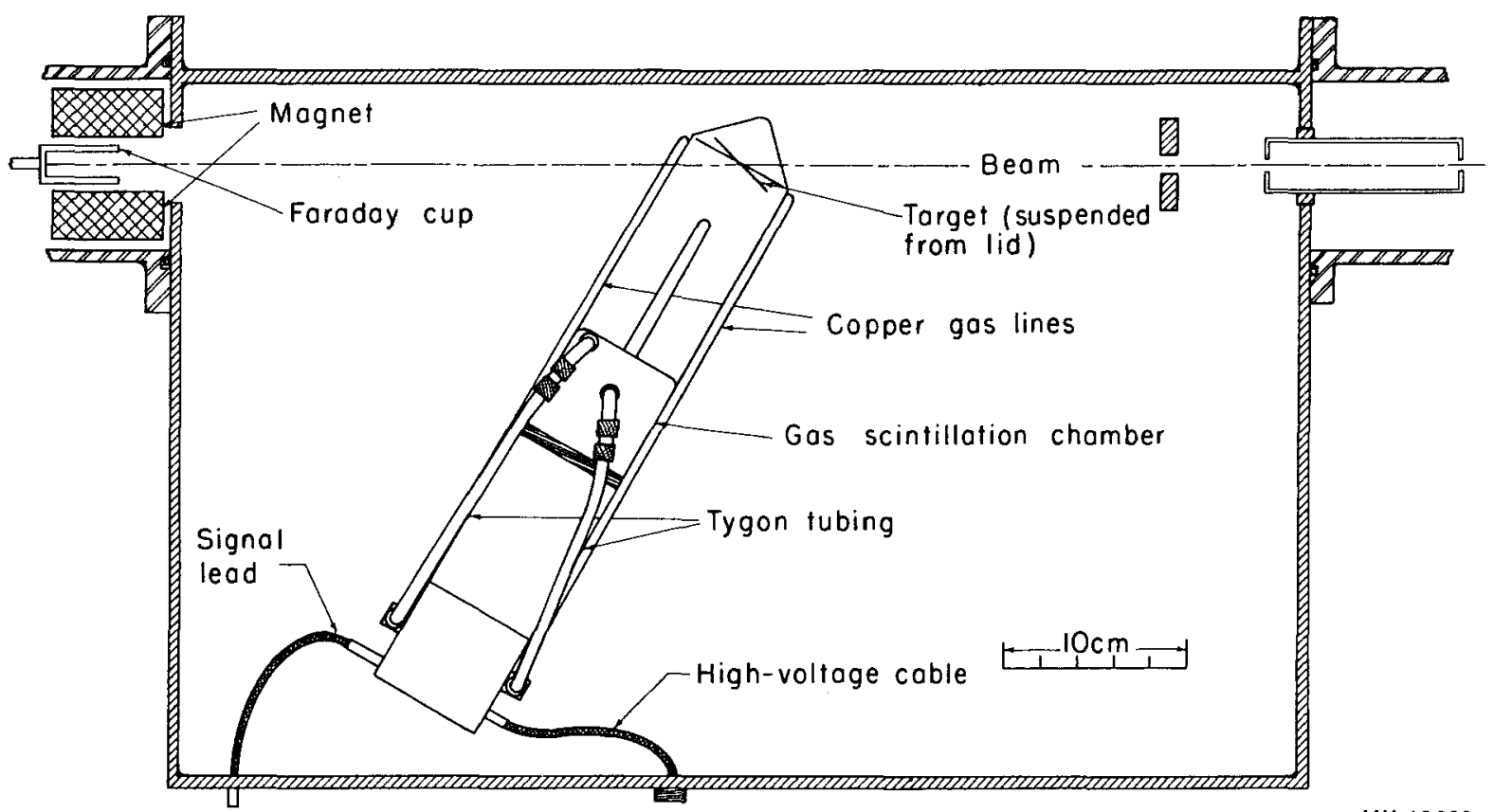

$\mathrm{MU}-19022$

Fig. 1. Schematic diagram of the vacuum tank. The gas scintillation chamber is shown in counting position. 
The solid-state detectors were made by diffusion of $n$ - or $p$-type impurities into one face of a silicon wafer containing an excess of the opposite type of impurity. 11,12 A more detailed account of the properties of these detectors is given elsewhere. 13

The electronic system used with the solid-state detectors is shown in Fig. 2. The same system was used with the gas scintillation chambers, except that pulses from the photomultiplier tube were fed directly into the cathode follower. The pulse generator was used to check the gain and noise level of the system and to make corrections for coincidence losses. A signal from the Hilac electronic system could be used to trigger the pulse generator during the $2-\mathrm{msec}$ bursts of particles.

A Cf $\mathrm{C}^{252}$ spontaneous fission sample was used to calibrate the detectors. A typical kinetic-energy spectrum of $\mathrm{Cf}^{252}$ fission fragments obtained with a solid-state detector is shown in Fig. 3. Energies corresponding to the peaks of the $\mathrm{Cf}^{252}$ spectrum were taken from the time-offlight data of Fraser and Milton. ${ }^{14}$ Corrections for energy loss in the detector windows were made with the help of the fragment range-energy data of Fulmer ${ }^{15}$ and Schmitt and Leachman. ${ }^{16}$ Corrections for self-absorption in the targets were determined empirically by bombardment of targets of various thicknesses.

Figure 4 shows a typical fragment-kinetic-energy spectrum obtained at 90 deg to the beam with a gold target bombarded with $93-\mathrm{Mev} \mathrm{C}^{12}$ ions. The large number of counts at the low-energy end of the spectrum resulted from pile-up of pulses produced by scattered beam particles and other light particles. Individual pulses from these light particles were clearly distinguishable from the pulses produced by fission fragments, because the sensitive counting regions of both types of counters could be made slightly longer than the range of the densely ionizing fission fragments. 

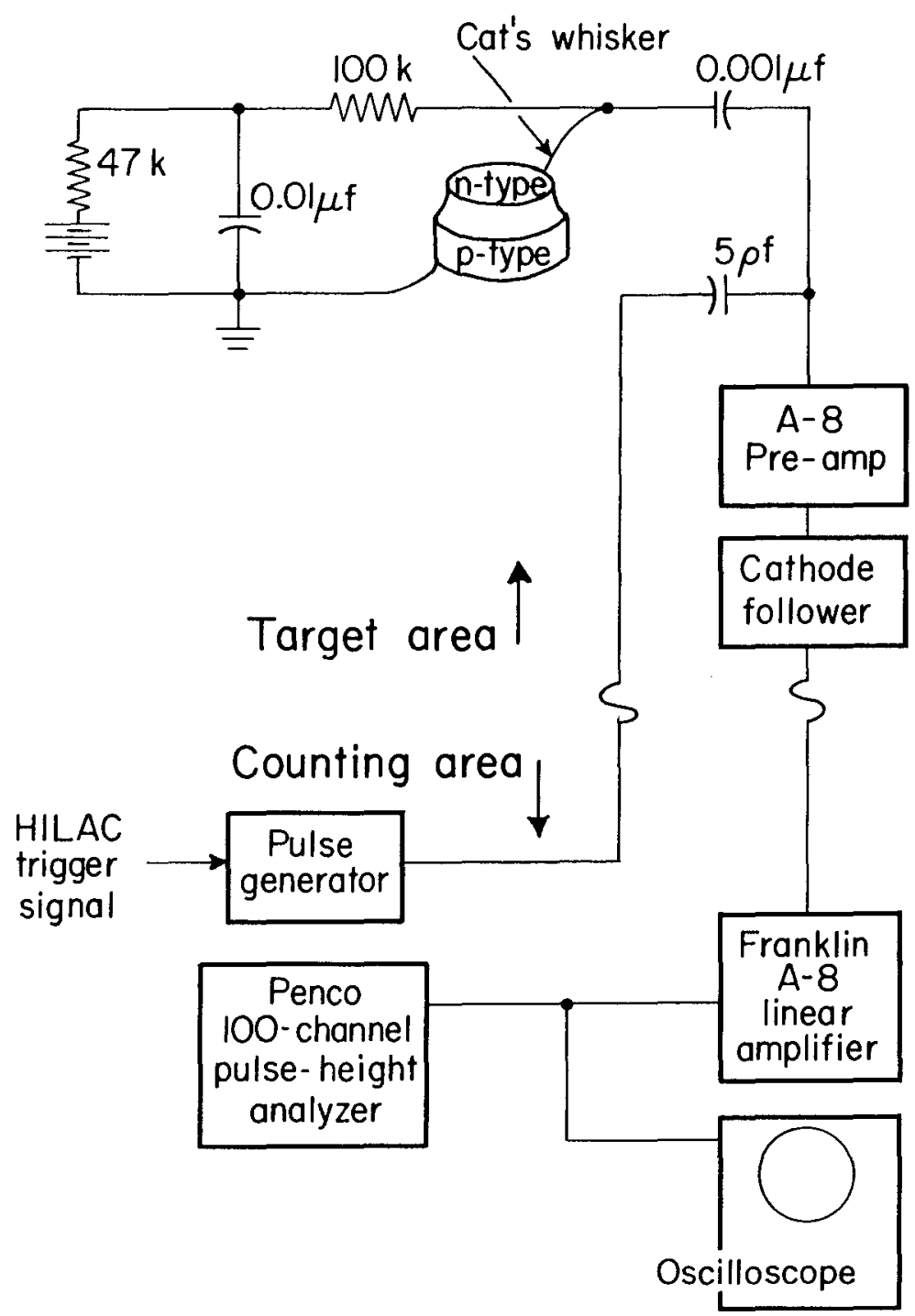

$M U-20123$

Fig. 2. Electronic system used with the solid-state detectors. 


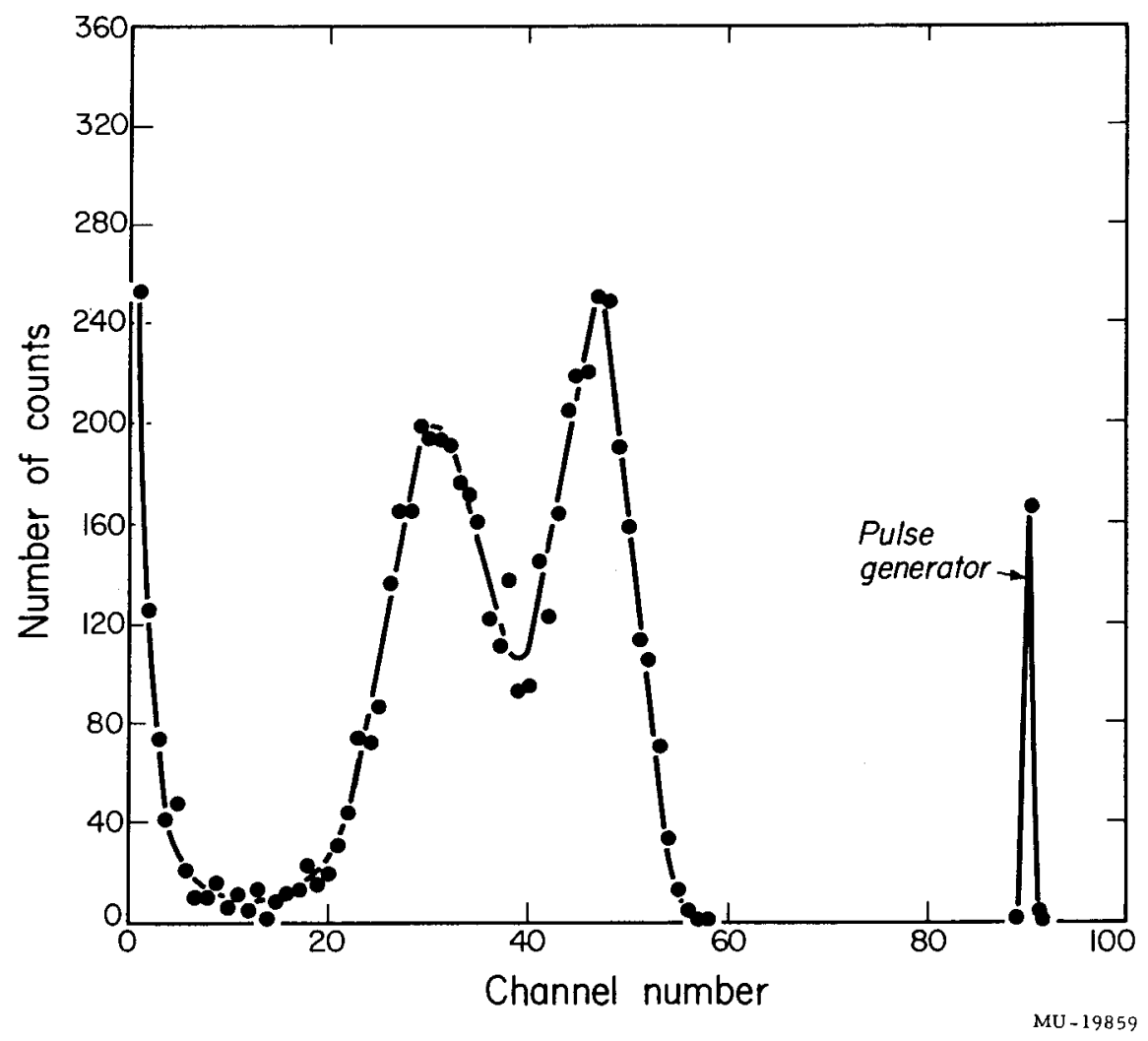

Fig. 3. Spectrum of fragment kinetic energies from spontaneous fission of $\mathrm{Cf}^{252}$. Observed with a solid-state detector reverse-biased by $9 \mathrm{v}$. 


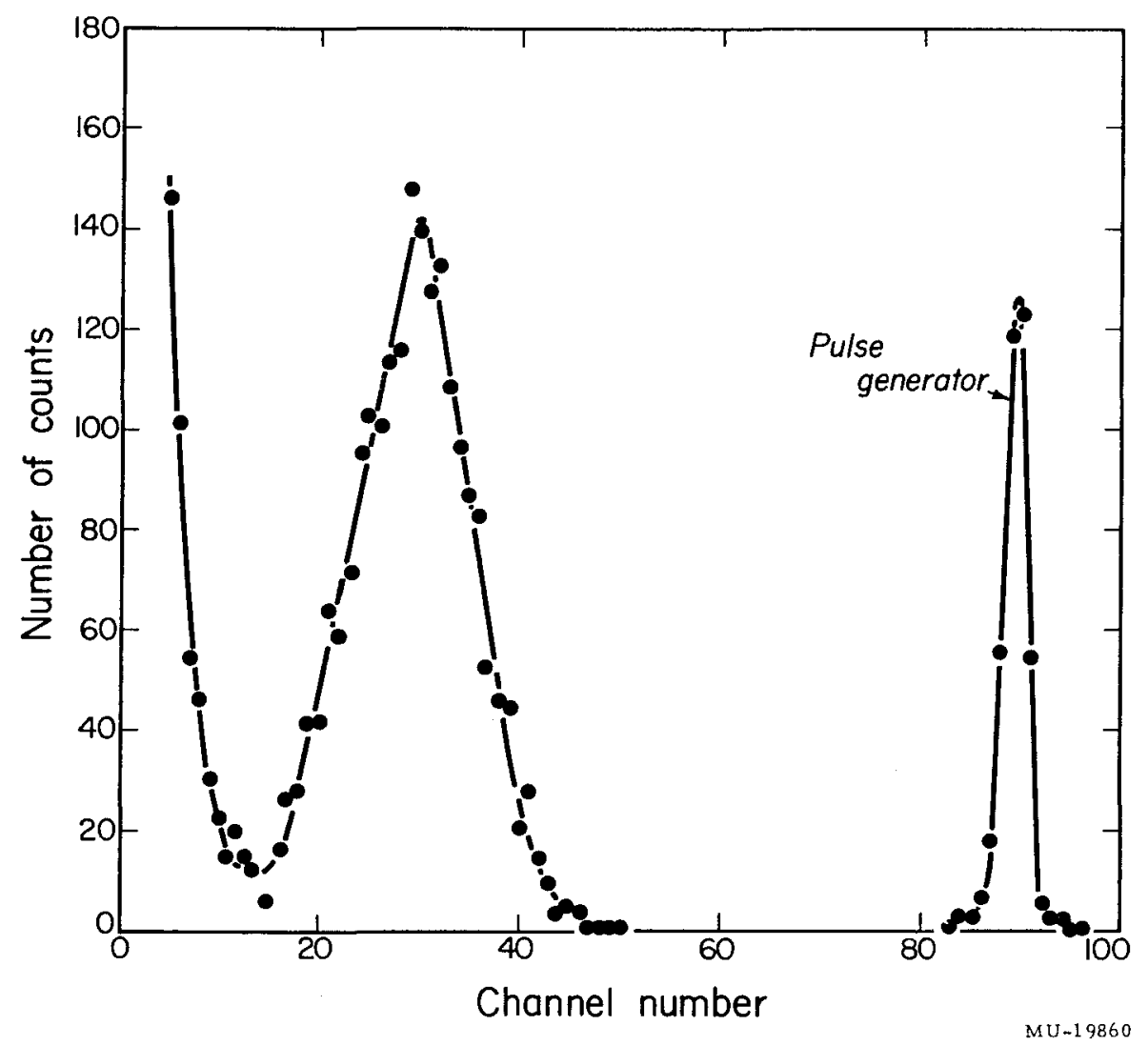

Fig. 4. Spectrum of fragment kinetic energies from fission of Au 197 induced by 93.3-Mev C 12 ions. Observed at 90 deg to the beam with a solid-state detector reverse-biased by $9 \mathrm{v}$. 
Thus, the lighter particles deposited only small amounts of energy in the counting region. However, "pile-up" of several of the small pulses in the electronic system could result in pulses of the size produced by the fission fragments. This difficulty became serious only at forward angles less than about $40 \mathrm{deg}$. At those angles, a log-arithmic subtraction of the pile-up background was often necessary. The gas scintillation chamber was mechanically limited to angles between 17 and $163 \mathrm{deg}$. The physically smaller solid-state detectors could be used between 8 and $172 \mathrm{deg}$. 


\section{EXPERIMENTAL RESULTS}

The fragment kinetic-energy spectra obtained at various angles to the beam were integrated, corrected for coincidence loss, and normalized to the same number of beam particles in order to obtain fragment angular distributions in the laboratory system. Two of the angular distributions are

shown in our earlier report. ${ }^{6}$ We have assumed that the peaks of the kineticenergy distributions represent the energy per fragment when symmetric division occurs. From the change in energy of the peak with laboratorysystem angle, we were able to obtain a value for the ratio $\eta$, given by $\eta=\frac{\text { velocity of the fissioning nucleus in the beam direction }}{\text { velocity of the fragment in the center-of-mass system }}$.

The values for $\eta$ obtained in this way are listed in Table I. These values have been used in transforming the laboratory-system angular distributions into the center-of-mass system. Within our limits of error, this procedure yields angular distributions that are symmetric about $90 \mathrm{deg}$ in the centerof-mass system. The resulting angular distributions obtained with 123.3-, 93.3-, and 72.4-Mev C ${ }^{12}$ ions on $\mathrm{Au}{ }^{197}$ are shown in Figs. 5 to 7 . The statistics on the points obtained at $72.4 \mathrm{Mev}$ are poor owing to the small fission cross section and large elastic-scattering cross sections at that energy。

Table I

\begin{tabular}{ccc}
\hline \multicolumn{3}{c}{ Values of the quantity $\eta$} \\
$\begin{array}{l}\text { Laboratory Energy } \\
\text { of carbon ion } \\
(\text { Mev })\end{array}$ & $\begin{array}{l}\text { From lab. energy } \\
\text { vs lab. angle }\end{array}$ & $\begin{array}{c}\text { For full momentum } \\
\text { transfer }\end{array}$ \\
\hline 123.3 & $0.223 \pm 0.01$ & 0.218 \\
93.3 & $0.188 \pm 0.01$ & 0.191 \\
72.4 & $0.164 \pm 0.01$ & 0.169 \\
\hline
\end{tabular}


UCR L-9257

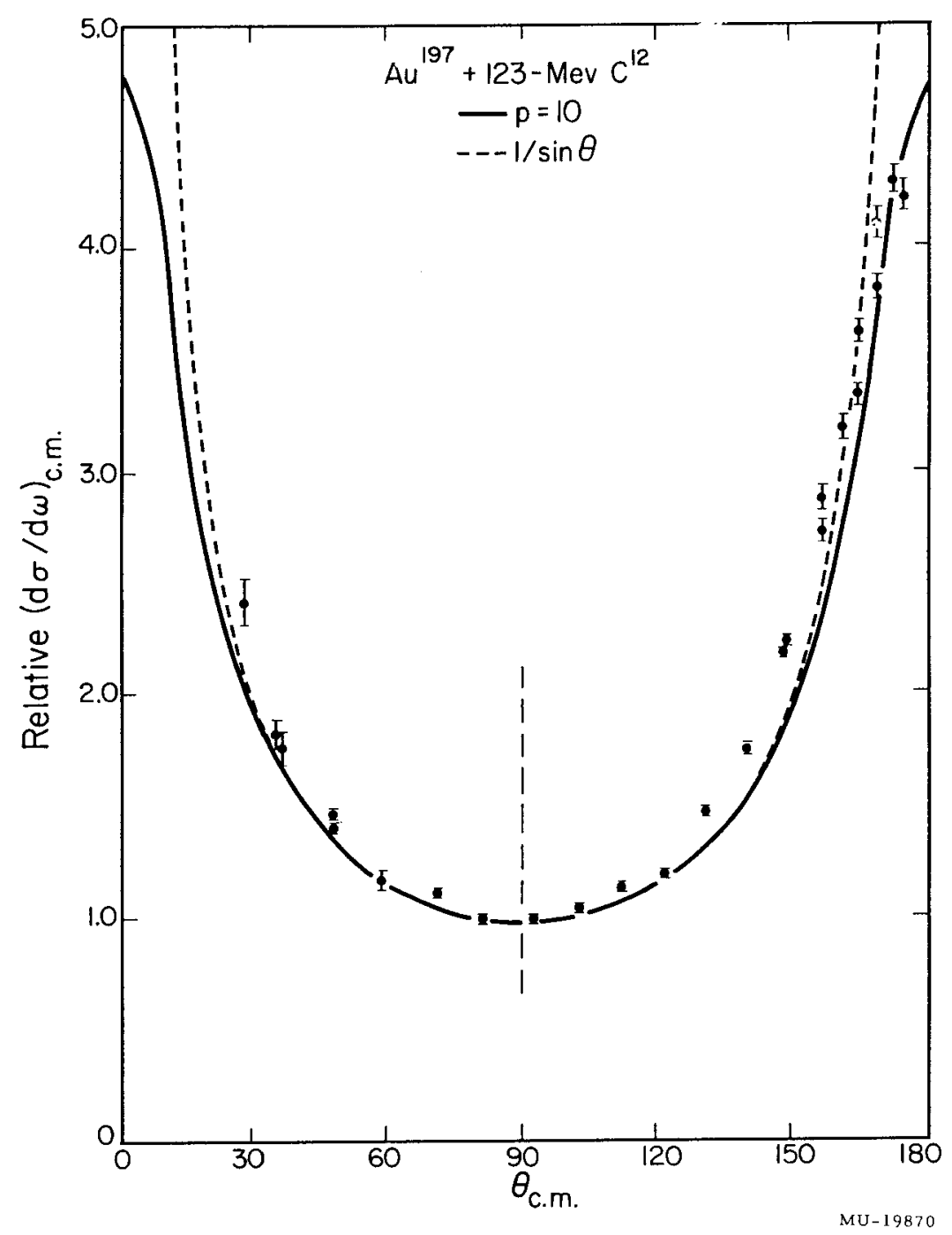

Fig. 5. Genter-of-mass angular distribution of fragments from fission of $\mathrm{Au} 197$ induced by $123.3-\mathrm{Mev} \mathrm{Cl2}$ ions. The solid curve represents Halpern and Strutinski's theoretical angular distribution with $p=10$ (see text). 


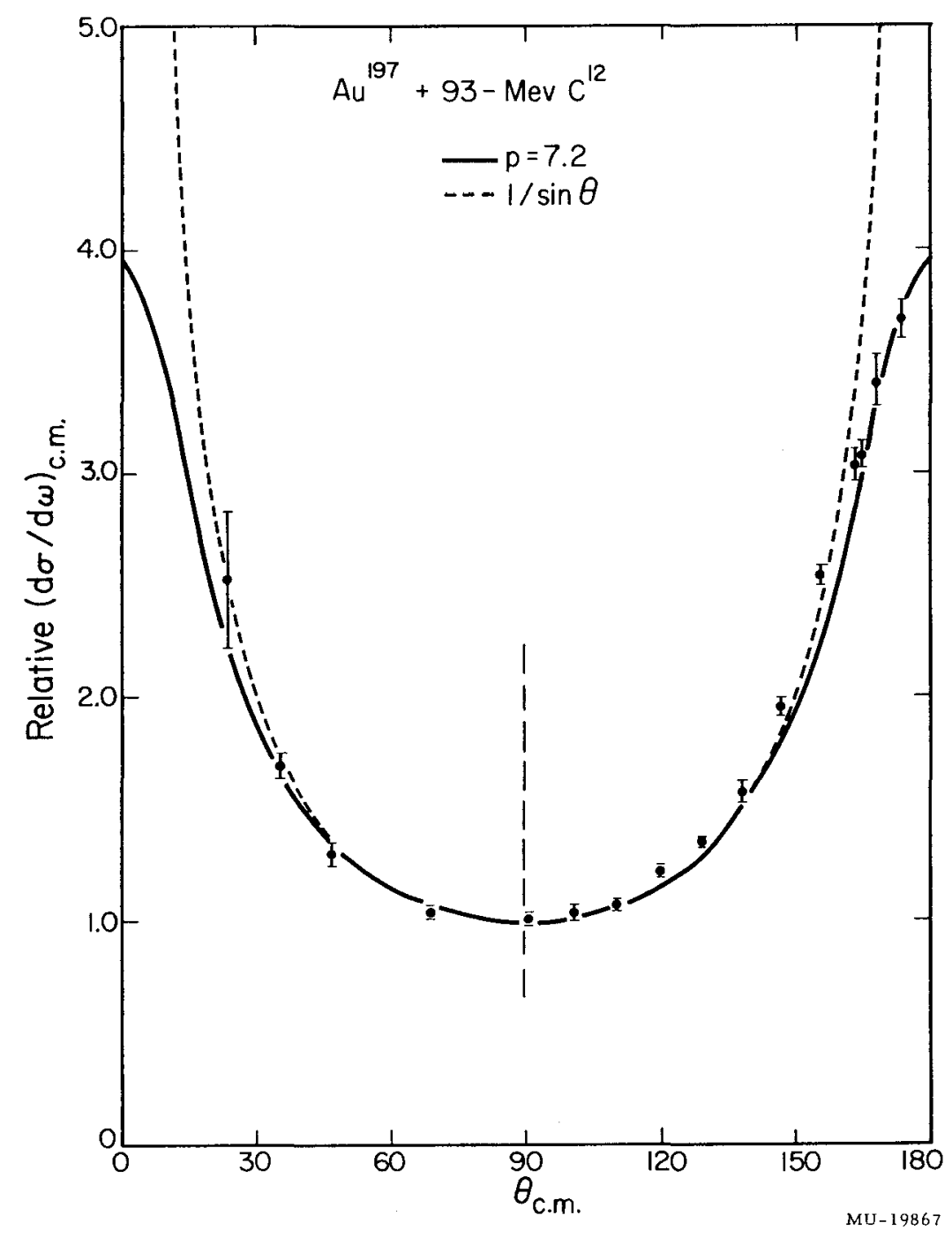

Fig. 6. Genter-of-mass angular distribution of fragments from fission of $\mathrm{Au} 197$ induced by $93.3-\mathrm{Mev} \mathrm{C}$ ions. The solid curve represents Halpern and Strutinski's theoretical angular distribution with $p=7.2$ (see text). 
UCRL-9257

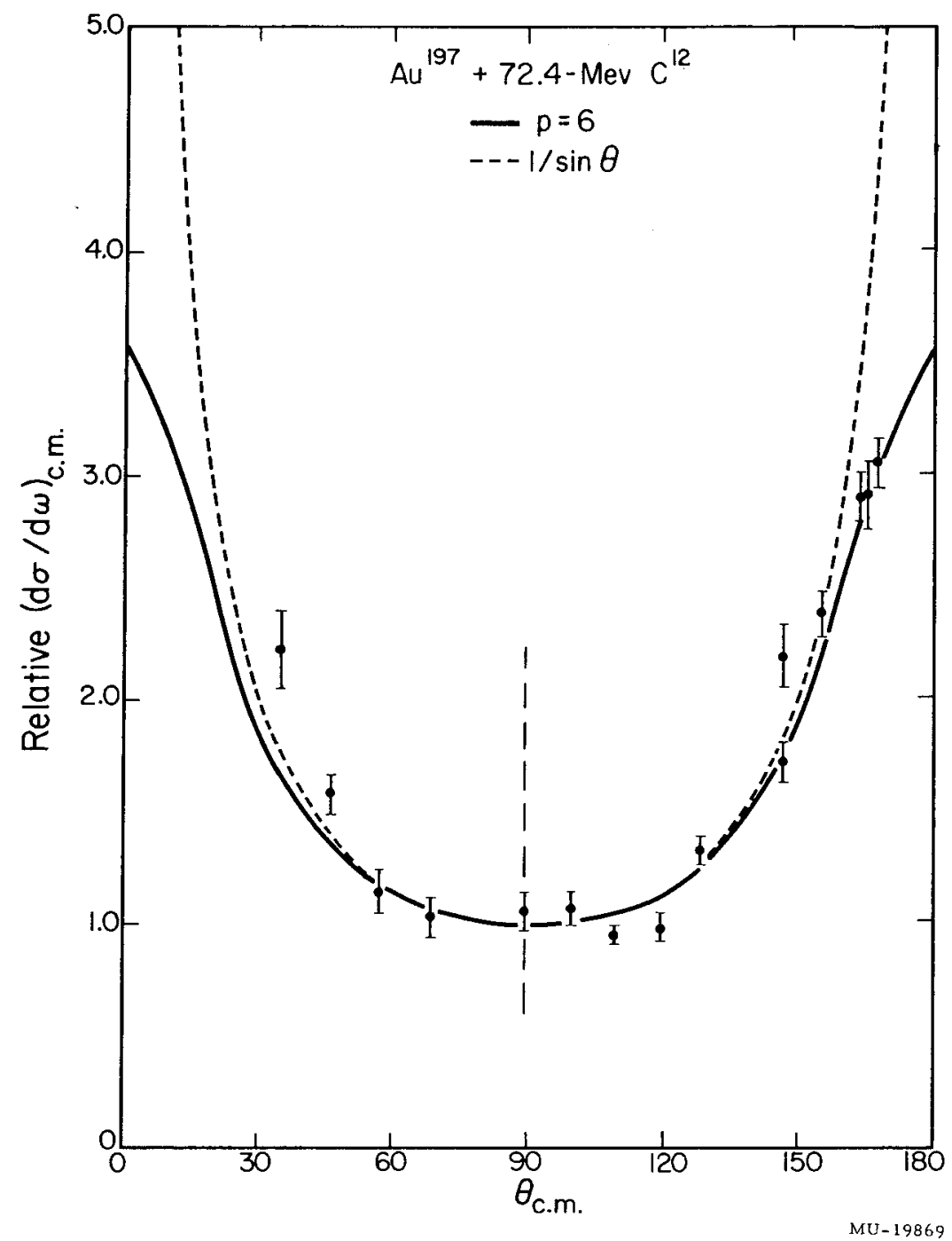

Fig. 7. Genter-of-mass angular distribution of fragments from fission of $\mathrm{Au} 197$ induced by $72.4-\mathrm{Mev} \mathrm{C}^{12}$ ions. The solid curve represents Halpern and Strutinski's theoretical angular distribution with $p=6$ (see text). 
Two types of experiments were done to obtain the fission cross sections as a function of bombarding energy. All fragment angular distributions were obtained at the energies indicated above. At closely spaced intervening energies, fragments were observed only at 90 deg to the beam. It is assumed that the integration factor from the angular-distribution experiments varies smoothly with bombarding energy. The absolute fission cross sections were obtained by relating the number of fragment counts to the number of elastically scattered carbon particles observed at small angles to the beam ( 30 to $60 \mathrm{deg})$. At these angles it is assumed that, for $72.4-\mathrm{Mev}$ $\mathrm{C}^{12}$ particles on gold, the scattering cross sections are equal to those calculated according to the Rutherford formula. The fission cross sections obtained by this procedure are shown in Fig. 8.

We have also obtained the most probable fission-fragment kinetic energy as a function of energy of the bombarding particle. This quantity increases from a value of $71 \pm 3 \mathrm{Mev}$ with $70-\mathrm{Mev} \mathrm{C}^{12}$ ions to $73 \pm 3 \mathrm{Mev}$ with $124-\mathrm{Mev} \mathrm{C}^{12}$ particles. With the assumption of symmetric binary fission, this result indicates a rise in total fragment kinetic energy release from $142 \pm 6$ to $146 \pm 6 \mathrm{Mev}$ over the range of bombarding energies. The values of the most probable fragment kinetic energy have been used to calculate the values of $\eta$ corresponding to full momentum transfer by the bombarding particle to the fissioning system. The results of these calculations, given in Table $I$, indicate that the $\eta$ values derived from the dependence of fragment kinetic energies on laboratory-system angle are consistent with full momentum transfer at all three bombarding energies. 


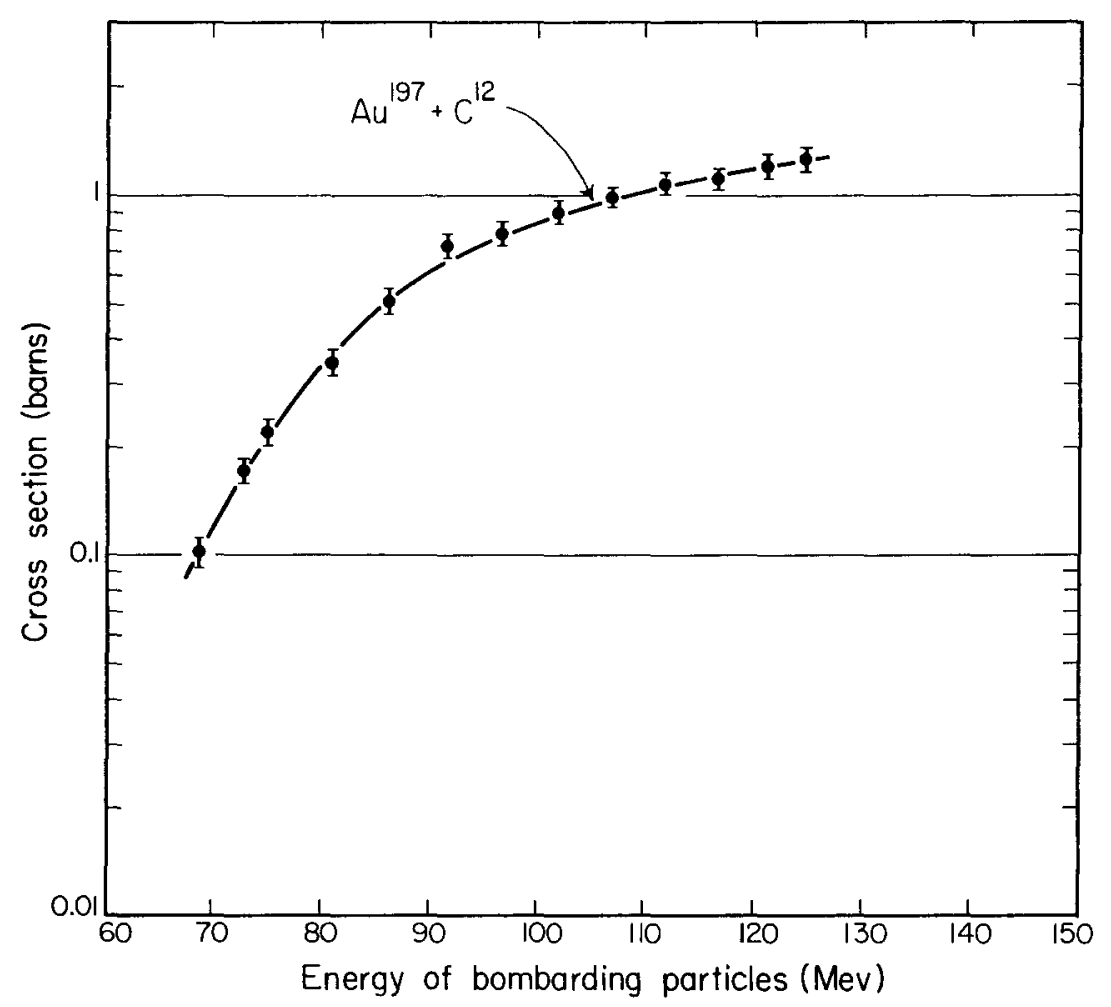

$M U-20107$

Fig. 8. Cross section for fission of $\mathrm{Au}^{197}$ induced by $\mathrm{C}^{12}$ ions as a function of bombarding energy. 


\section{INTER PRETATION OF RESULTS}

The fragment angular distributions have been compared with theoretical curves calculated according to the treatments by Griffin ${ }^{3}$ and by Halpern and Strutinski。 ${ }^{1}$ In the region between approximately 105 and 160 deg (and the corresponding forward angles), the points obtained with 123.3-Mev C $\mathrm{C}^{12}$ particles on gold lie slightly above the $1 / \sin \theta$ curve. This feature is in agreement with the Griffin curves. Near $180 \mathrm{deg}$, the experimental angular distributions exhibit some tendencies toward the curvature predicted by Halpern and Strutinski, rather than the linear shape given by Griffin $\sim$ s equations. With our limits of error, it is not possible to rule out either of the theoretical treatment. However, this is not too important as there is no fundamental difference between them, and the end results--the derived excitation energies at the time of fission--are nearly the same regardless of the treatment used. We have used Halpern and Strutinski's method in our interpretations because it should be more applicable to the large angular momenta and excitation energies present in the compound nuclei that were formed in our experiments.

In Figs。 5 to 7 , we have shown the Halpern-and-Strutinski curves that best fit the experimental data. The parameter $p$ is equal to $I_{\mathrm{m}}^{2} / 4 \mathrm{~K}_{0}^{2}$ where $I_{m}$ is approximately the maximum angular momentum of the compound nuclei, and $K_{0}$ is the mean value of the projection of the angular momentum of the fissioning nucleus along the direction of the separating fission fragments (see Ref. 1). We have estimated $I_{m}^{2}$ from the compoundnucleus-formation calculations of Thomas, assuming the square-well potential with a radius parameter of $1.5 \times 10^{-13} \mathrm{~cm} .^{17}$ In accord with Halpern and Strutinski, we assure that there is no change in the angular momentum of the compound nucleus if small particles are evaporated prior to fission. 
From the resulting value of $\mathrm{K}_{0}^{2}$ and the curve of Ref. 1 (Fig. 2), we determine the value of the quantity $\left(E_{e x}-E_{f}\right)$, where $E_{e x}$ is the excitation energy of the fissioning nucleus, and $E_{f}$ is the height of the fission barrier. The fission-barrier heights have been estimated by using the equations of Pik-Pichak ${ }^{18}$ and Hiskes ${ }^{19}$ for fission of rotating nuclei. In this framework, the $f$ Ission-barrier height is equal to the energy difference between the stable rotating nucleus and the fissioning nucleus at the saddle point. The calculated fission barriers are of the order of 10 to $15 \mathrm{Mev}$ and seem reasonable when compared to those calculated according to Swiatecki's method that involves the difference between actual ground-state masses and a smooth mass surface. 20 The results of this analysis of the angular-distribution data are given in Table II.

From the results presented in Table II, it would appear that fission occurs at about the same average excitation energy regardless of the excitation energy of the original compound nucleus. This observation implies the evaporation of a higher average number of particles prior to fission with increasing bombarding energy. For example, at the highest bombarding energy, this treatment of the angular-distribution data indicates that the following sequences of particles could be emitted before reaching the average fissioning nucleus: $7 n ; p 6 n ; a 5 n ; p a 4 n ; 2 a 3 n$. These results are consistent with Blann's radiochemical mass-yield data for fission of gold with carbon ions. 21 
Table II

Quantities obtained from the angular-distribution data for fission of gold with carbon ions.

\begin{tabular}{|c|c|c|c|c|c|c|c|}
\hline $\begin{array}{l}E_{C^{12}}^{12} \\
(\mathrm{Mev})\end{array}$ & $\begin{array}{l}\mathrm{E}_{\text {ex }} \text { of } \\
\text { initial } \\
\text { comp. nucl. } \\
\text { (Mev) }\end{array}$ & $\mathrm{P}$ & $\mathrm{I}_{\mathrm{m}}^{2}$ & $\mathrm{~K}_{0}^{2}$ & $\begin{array}{c}E_{\mathrm{ex}}-E_{f} \\
(\mathrm{Mev})\end{array}$ & $\begin{array}{c}\text { Calc. }^{a} \\
E_{f} \\
(\mathrm{Mev})\end{array}$ & $\begin{array}{c}\mathrm{E}_{\mathrm{ex}} \\
(\mathrm{Mev})\end{array}$ \\
\hline 123.3 & 98.6 & 10 & 4600 & 115 & 13.5 & $8-13$ & $21.5-26.5$ \\
\hline 93.3 & 71.4 & 7.2 & 2530 & 87.8 & 12 & $10-16$ & $22-28$ \\
\hline 72.4 & 51.6 & 6 & 1118 & 46.6 & 9 & $11-14$ & $20-23$ \\
\hline
\end{tabular}

${ }^{a}$ Calculated according to Refs. 18 and 19. Includes lowering of barrier due to rotation. Upper and lower limits refer to nuclei having the lowest and highest values of $\mathrm{Z}^{2} / \mathrm{A}$ energetically obtainable by emission of particles prior to reaching the observed value of $E_{e x}-E_{f}$.

Unfortunately, there are two possible effects that make this interpretation somewhat uncertain. According to both theoretical treatments, the fission probability is independent of the angular momentum of a nucleus; ${ }^{1}, 3$ however, Pik-Pichak's calculations indicate that $\Gamma_{\mathrm{f}} / \Gamma_{\mathrm{n}}$ is an increasing function of angular momentum. ${ }^{18}$ If this is true, fission takes place with higher relative probability from the high-spin nuclei than from those in low-spin states. Thus, the average angular momentum of the fissioning nuclei may be considerably larger than the average angular momentum of all the compound nuclei. Also, j.t has been suggested that the value of $K_{0}^{2}$ for a given value of $\left(E_{e x}-E_{f}\right)$ may be lower in the astatine region than among the heavier elements. ${ }^{22}$ Such an effect could arise from the influence of the closed shells of 82 protons and 126 neutrons. Both effects would tend to cause the angular distributions to be more anisotropic than 
predicted strictly by the Halpern and Strutinski model. Consequently, this would yield values for $\left(E_{e x}-E_{f}\right)$ that are too small. This trend would tend to cancel any errors introduced as a result of the assumption that the angular momentum of the compound nucleus is not reduced by evaporation of particles prior to fission.

To obtain information about the probability of fission, we have divided the fission cross sections shown in Fig. 8 by the cross sections for compound-nucleus formation, $\sigma$ comp' calculated by using a square-well nuclear potential with a radius parameter of $1.5 \times 10^{-13} \mathrm{~cm} .^{17}$ The resulting values of $\sigma_{f} / \sigma_{\text {comp }}$ are plotted as a function of excitation energy of the initial compound nucleus in Fig. 9. If $\Gamma_{\mathrm{f}} / \Gamma_{\mathrm{T}}\left(\Gamma_{\mathrm{T}}\right.$ is the total level width) remained constant with increasing excitation energy, $\sigma_{\mathrm{f}} / \sigma_{\text {comp }}$ would increase at the higher energies because there are more stages in the chain of de-exciting nuclei at which fission could compete with other decay modes. From the experimental results, one can infer that above about $70 \mathrm{Mev}, \Gamma_{\mathrm{f}} / \Gamma_{\mathrm{T}}$ decreases with increasing excitation energy. This observation is at least in qualitative agreement with the results of the angular distribution experiments。

A note of caution should be added. It is certainly not clear that the calculation cross sections for compound-nucleus formation are correct. Whereas the $\eta$ values that we obtain for fission of gold with carbon ions are consistent with the concept of formation of a compound nucleus, preliminary measurements in the uranium-plus-carbon system indicate that, at higher bombarding energies $(\geqslant 90 \mathrm{Mev})$, the average forward momentum of the fissioning system is considerably less than that of the bombarding particle. 23 Such an effect could arise if fission were, in some cases, the result of reactions in which not all of the bombarding particle entered 


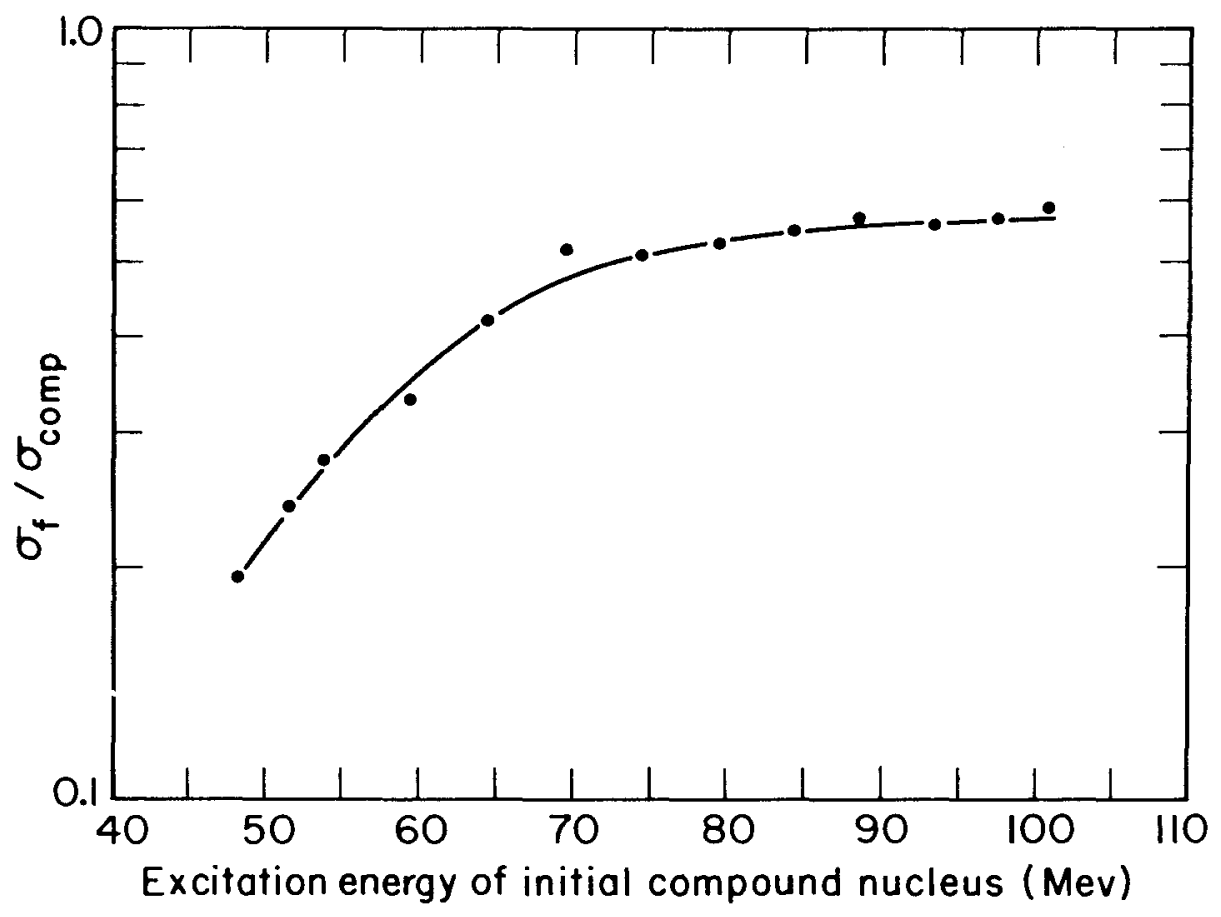

$\mathrm{MU}-20469$

Fig. 9. Reduced fission cross section $\left(\sigma_{f} / \sigma_{\text {comp }}\right.$ ) for Au 197 bombarded with $C^{12}$ ions as a function of excitation energy of the initial compound nucleus. 
the target nucleus. On the other hand, the total fission cross sections for uranium (but not gold) at bombarding energies between 70 and $124 \mathrm{Mev}$ are in agreement with the calculated cross sections for compound-nucleus formation. These two observations suggest that the cross sections calculated for the formation of compound nuclei (by using the square-well nuclear potential with radius parameter of $1.5 \times 10^{-13} \mathrm{~cm}^{17}$ ) are larger than the observed cross sections for fission by compound nuclei. Although in non-compound-nucleus reactions excitation energy sufficient to cause fission may be deposited in the nucleus formed by bombardment of uranium the intermediate state formed in these reactions with gold targets may not have enough excitation energy or sufficiently large atomic number to undergo fission with appreciable probability. From the results of a study of fission of $\mathrm{Bi}^{209}$ carbon and oxygen ions induced by Britt and Quinton have also suggested that the calculated cross sections for the formation of compound nuclei are too high. 24

The interpretations of the results of the angular-distribution and cross-section measurements suggest that fission resulting from carbon bombardment of gold occurs at low excitation energies following the emission of several nucleons. Three possible explanations for the apparent decrease in fission probability at high excitation energies are:

(a) Charged-particle emission. One expects the competition from charged-particle evaporation to increase rapidly with excitation energy. 25 This effect has been observed by Quinton et al., who found a ratio of approximately two alpha particles (in addition to protons) per three fission events when $\mathrm{Au}^{197}$ was bombarded with $160-\mathrm{Mev} 0^{16}$ ions. 26 
(b) Mass-number dependence of fission probability. Many authors have observed an increase in $\Gamma_{f} / \Gamma_{n}$ with decreasing mass number for a given atomic number..$^{27-30}$ In work not reported here, we have measured the fission cross sections of platinum (enriched in $\mathrm{Pt}^{198}$ ) bombarded with nitrogen ions to produce an initial compound nucleus of $\mathrm{At}^{212}$. For the same initial excitation energy, the value of $\sigma_{\mathrm{f}} / \sigma_{\text {comp }}$ is lower than the corresponding value in the gold-plus-carbon system, in which the initial compound nucleus, $\mathrm{At}^{209}$, is three neutrons lighter. Also, the cross sections for neutron-evaporation reactions decrease in going from the compound nucleus $\mathrm{At}^{212}$ to $\mathrm{At}^{210}$ (from $\mathrm{Pt}^{196}+\mathrm{N}^{14}$ ) to $\mathrm{At}^{209}{ }^{31}$ Both trends are in agreement with the notion of increasing relative fission probability with decreasing mass number of the compound nucleus.

(c) Angular momentum and level densities. The calculations of Ericson and Strutinski indicate that at some given excitation energy, $E_{\text {ex }}$, the density of levels of spin $\not{\mathscr{N}}$ is proportional to $(2 j+1) \exp \left[\hbar^{2}-j(j+1) / 2 \mathrm{JT} 1\right.$, where $J$ is the moment of inertia, and $T$ is the nuclear temperature, which is given by $\left(10 \mathrm{E}_{\mathrm{ex}} / \mathrm{A}\right)^{1 / 2}$. 32 At very low excitation energies, this model predicts small probabilities for states with spins of the order of the average value of those formed in the initial interaction between the bombarding particle and the target nucleus. In the early stages of evaporation of particles from the compound nucleus, the excitation energy, and thus the density of high-spin states, is large. Therefore, it is expected that in early stages of the evaporation process, the average angular momentum of the compound nucleus may be reduced by only small amounts. ${ }^{33}$ Thus it is possible that particle evaporation may proceed to an excitation energy only slightly above the neutron binding energy while retaining most of the angular momentum of the initial compound nucleus. Emission of neutron will be greatly hindered 
because of the small density of high-spin states in the residual nucleus and the decreasing probability for transmission of neutrons through the nuclear surface with higher orbital angular momentum. ${ }^{34}$ Because of the hindrance of neutron emission in the final stages of de-excitation, the probability for fission (a process in which large amounts of angular momentum can be easily carried off) may be greatly enhanced over its value for low-spin states, other conditions being the same. Pik-Pichak's predicted decrease in fission barriers with increasing angular momentum would also contribute to this trend. 18

We feel that these three trends produced the observed results of fission at low excitation energies. However, it is not possible, on the basis of our results alone, to determine the relative importance of the three factors.

The value obtained for the total fragment kinetic-energy release agrees well with Terrell's correlation of energy release with $z^{2} / A^{1 / 3}$ of the fissioning nucleus. ${ }^{35}$ The angular-distribution experiments indicate that the average excitation energy at which fission occurs is approximately independent of bombarding energy. Thus, our data do not provide a check on the variation of kinetic-energy release with excitation energy of the fissioning nucleus. The slight increase in kinetic-energy release with bombarding energy is apparently the result of emission of a larger average number of neutrons prior to fission. Thus, fission at higher bombarding energies occurs from nuclei that are more neutron-deficient and have larger values of the quantity $\mathrm{z}^{2} / \mathrm{A}^{1 / 3}$. 


\section{SUMMARY}

In summary, we find that the shapes of the theoretical angular distributions predicted by Griffin ${ }^{3}$ and Halpern and Strutinski ${ }^{1}$ agree well with those of the fragment angular distributions that we have determined for fission of gold with carbon ions. It should be noted, however, that for gold bombarded with $123.3-\mathrm{Mev} \mathrm{C}^{12}$, the points between approximately 105 and $160 \mathrm{deg}$ (and the corresponding forward angles) in the center-of-mass system lie slightly above a $1 / \sin \theta$ curve. This is in better agreement with Griffin's predictions than those of Halpern and Strutinski.

The anisotropies of the angular distributions and the fission cross sections as functions of bombarding energy are consistent with the occurrence of fission at low average excitation energies (about $25 \mathrm{Mev}$ ) following evaporation of particles from the initial compound nucleus. This observation is explained on the basis of (a) an increase in probability for charged-particle evaporation with increasing excitation energy, (b) an increase in relative fission probability with decreasing mass number for a given atomic number, and (c) hindrance of neutron evaporation at low excitation energies as a result of angular-momentum and level-density effects. Our data do not suffice to determine the relative importance of the three effects.

At all bombarding energies, we find that for reactions that lead to fission, the full momentum of the carbon ion is transferred to the gold target nucleus. From this result, it is inferred that fission results only from reactions in which a compound nucleus is formed from the carbon particle and the gold nucleus. This observation is in contrast to those in the uranium-plus-carbon system, in which, at higher bombarding energies $(\geqslant 90 \mathrm{Mev})$, there is incomplete momentum transfer in some of the reactions that lead to fission. 23 
The slight increase in fragment kinetic-energy release with increasing bombarding energy is attributed to an increase in the average number of neutrons evaporated prior to fission. Thus, the fissioning nuclei produced in the higher-energy bombardments have higher values of the quantity $\mathrm{Z}^{2} / \mathrm{A}^{1 / 3}$ and would be expected, on the basis of Terrell's correlation of kinetic energy release with $z^{2} / A^{1 / 3}, 35$ to yield higher kinetic energies.

\section{ACKNOW LEDGMEN TS}

The authors wish to thank Robert Latimer for his assistance in the cross-section experiments. We would also like to acknowledge the assistance of the Hilac crew. 


\section{REFERENCES}

1. A. Bohr, in Proceedings of the International Conference on the Peaceful Uses of Atomic Energy, Geneva, 1956 (United Nations, New York, 1956), Vol. 2, p. 151 .

2. J. J. Griffin, Phys. Rev. 116, 107 (1959).

3. I. Halpern and V. Strutinski, in Proceedings of the Second International Conference on the Peaceful Uses of Atomic Energy, Geneva, 1958 (United Nations, New York 1958), Vol。15, p. 408.

4. C. T. Coffin and I. Halpern, Phys。Rev。 112, 536 (1958).

5. A. W. Fairhall, R. C. Jensen, and E. F. Neuzil, in Proceedings of the Second International Conference on the Peaceful Uses of Atomic Energy, Geneva, 1958 (United Nations, New York, 1958), Vol. 15, p. 452.

6. Glen E. Gordon, Almon E. Larsh, and Torbjorn Sikkeland, Phys. Rev. (in press).

7. John R. Walton, Lawrence Radiation Laboratory, University of California, unpublished data, 1959.

8. A. Sayres and C.S. Wu, Rev, Sci. Instr。 28, 758 (1958).

9. C. M. Huddleston, in Proceedings of the Second International Conference on the Peaceful Uses of Atomic Energy, Geneva, 1958 (United Nations, New York 1958), Vol. 14, p. 298.

10. R. A. Nobles and R. B. Leachman, Nuclear Phys. 5, 211 (1958).

11. William Hansen, Lawrence Radiation Laboratory, University of California, private communication, 1959.

12. S. S. Friedland, J. W. Mayer, and J。 S. Wiggins, Nucleonics 18, No. 2, $54(1960)$. 
13. Almon E. Larsh, Glen E. Gordon, and Torbjorn Sikkeland, Use of Silicon $\mathrm{p}-\mathrm{n}$ Junction Detectors in Studies of Nuclear Reactions Induced by Heavy Ions, UCRL-9240, May 1960 (to be published).

14. J. S. Fraser and J. C. D. Milton, in Proceedings of the Second International Conference on the Peaceful Uses of Atomic Energy, Geneva, 1958 (United Nations, New York, 1958), Vol. 15. p. 216.

15. Clyde Fulmer, Phys. Rev. 108, 1113 (1957).

16. H. W. Schmitt and R. B. Leachman, Phys.Rev。102, 183 (1956).

17. T. D. Thomas, Phys. Rev。116, 703 (1959).

18. G. A. Pik-Pichak, Soviet Phys. J.E.T.P. ㄱ, 238 (1958).

19. John Hiskes, Lawrence Radiation Laboratory, University of California, private communication, 1960 .

20. W. J. Swiatecki, Lawrence Radiation Laboratory, University of California, private communication. The fission-barrier calculations are similar to those used in the systematics of spontaneous-fission half lives, W. J. Swiatecki, Phys。Rev。100, 937 (1955).

21. H. Marshall Blann, Fission of Gold with $112-\mathrm{Mev} \mathrm{C}^{12}$ Ions:

A Yield-Mass and Charge-Distribution Study (Thesis), UCRL-9190, May 1960 (unpublished).

22. $\dot{R}$. Vandenbosch and $J$. R. Huizenga, Nuclear Fission (to be published in Nuclear Reactions (North-Holland Publishing Co., Amsterdam, Holland) Vol。2.

23. Almon E. Larsh, Glen E. Gordon, Torbjorn Sikkeland, and John R. Walton, Heavy-Ion-Induced Nuclear Fission, (to be published in Proc. Second Gatlinburg Conf. on Reactions between Complex Nuclei, May, 1960). 
24. H. C. Britt and A. R. Quinton, Fission Studies with Carbon and Oxygen Beams, (to be published in Proc. Second Gatlinburg Conf. on Reactions between Complex Nuclei, May 1960).

25. Doestrovsky, Z. Fraenkel, and G. Friedlander, Phys.Rev。 116,683 (1959).

26. A. R. Quinton, H. C. Britt, W. J. Knox, and C. E. Anderson, The Fission of Gold by Oxygen Nuclei, Yale University preprint, 1960 (to be published).

27. R. H. Goeckermann and I. Perlman, Phys. Rev. 76, 628 (1949).

28. R. Vandenbosch and G. T. Seaborg, Phys. Rev。110, 507 (1958).

29. R. Vandenbosch and J. R. Huizenga, in Proceedings of the Second International Confernece on the Peaceful Uses of Atomic Energy, Geneva, 1958 (United Nations, New York, 1958), Vol.15, p.284. Most of the available data on fissionabilities are summarized in this paper and in Ref. 22 .

30. A. W. Fairhall and E. F. Neuzil, University of Washington, (to be published). Data and analysis of cross sections for fission of lead isotopes with helium ions are given by I. Halpern, in Ann. Revs. Nuclear Sci. 9, 245 (1959).

31. T. D. Thomas, R. M. Latimer, G. E. Gordon, and G. T. Seaborg, Lawrence Radiation Laboratory, University of California, unpublished data, 1959 .

32. T. Ericson and V. Strutinski, Nuclear Phys。 8, 284 (1958).

33. T. Darrah Thomas, Brookhaven National Laboratory, private communication, 1960. Preliminary calculations by Thomas based on the Ericson-Strutinski level-density expressions indicate that the first neutron emitted from an $\mathrm{At}^{209}$ nucleus excited to $99 \mathrm{Mev}$ and having $66 \hbar$ of angular momentum reduces the average spin value by about 1 unit of $\hbar$. It should be noted that in this calculation, the value of $J$ was assumed to be equal 
to the rigid-body moment of inertia of the nucleus.

34. J. M. Blatt and V. F. Weisskopf, Theoretical Nuclear Physics, (John Wiley and Sons, New York, 1952).

35. James Terrell, Phys. Rev. 113, 527 (1959). 

This report was prepared as an account of Government sponsored work. Neither the United States, nor the Commission, nor any person acting on behalf of the Commission:

A. Makes any warranty or representation, expressed or implied, with respect to the accuracy, completeness, or usefulness of the information contained in this report, or that the use of any information, apparatus, method, or process disclosed in this report. may not infringe privately owned rights; or

B. Assumes any liabilities with respect to the use of, or for damages resulting from the use of any information, apparatus, method, or process disclosed in this report.

As used in the above, "person acting on behalf of the Commission" includes any employee or contractor of the Commission, or employee of such contractor, to the extent that such employee or contractor of the Commission, or employee of such contractor prepares, disseminates, or provides access to, any information pursuant to his employment or contract with the Commission, or his employment with such contractor. 